Methods We searched the Pubmed database for prospective cohort and nested case-control studies of whole grain intake and risk of incident colorectal cancer, up to December 2010. Summary RRs were calculated using a random effects model.

Results Seven cohort studies reported results for total whole grain intake and colorectal cancer risk. The summary RR for high vs low intake of whole grain was 0.79 (95\% CI 0.72 to 0.86$)$, with no significant heterogeneity, $\mathrm{I}^{2}=0 \%$. The summary RR for a 3 servings per day increment was 0.81 (95\% CI 0.75 to 0.88 ), with little heterogeneity, $\mathrm{I}^{2}=15 \%$. A similar reduction in risk was also found for colon cancer (summary $\mathrm{RR}=0.81,95 \% \mathrm{CI} 0.70$ to $0.95, \mathrm{I}^{2}=0 \%$ ), but the result for rectal cancer was not statistically significant and there was substantial heterogeneity (summary $\mathrm{RR}=0.75,95 \% \mathrm{CI} 0.53$ to $1.08, \mathrm{I}^{2}=87 \%$ ).

Conclusion Our results support the hypothesis that whole grain consumption protects against colorectal cancer.

\section{P2-19 MOBILE PHONE USE AND LOCATION OF GLIOMA: A CASE- CASE ANALYSIS}

doi:10.1136/jech.2011.142976h.56

\begin{abstract}
${ }^{1,2} \mathrm{~A}$ Auvinen, ${ }^{*}{ }^{2} \mathrm{~S}$ Larjavaara, ${ }^{3} \mathrm{~T}$ Tynes, ${ }^{4} \mathrm{~J}$ Schuz, ${ }^{5} \mathrm{~A}$ Swerdlow, ${ }^{6} \mathrm{M}$ Feychting, ${ }^{7} \mathrm{C}$ Johansen, ${ }^{8} \mathrm{~S}$ Lagorio. ${ }^{1}$ STUK - Radiation and Nuclear Safety Authority, Helsinki, Finland; ${ }^{2}$ University of Tampere, Tampere, Finland; ${ }^{3}$ Norwegian Radiation Protection

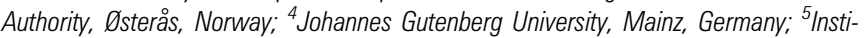
tute of Cancer Research, Surrey, UK; ${ }^{6}$ Karolinska Institute, Stockholm, Sweden; ${ }^{7}$ Danish Cancer Society, Copenhagen, Denmark; ${ }^{8}$ Istituto Superiore di Sanita, Rome, Italy
\end{abstract}

Introduction The risk of brain tumours related to mobile phone use has been assessed in several studies without conclusive results. As the radiofrequency field emitted by the phone depends strongly on the distance from the source, any effect should be highly localised and occur mainly in the part of the brain absorbing most of the energy. We assessed the distance from the exposure source (typical location of mobile phone when in use) and the mid-point of the glioma recorded in blinded fashion from radiologic images.

Methods A total of 888 cases aged 18-69 years from seven European centers (Denmark, Finland, Germany, Italy, Norway, Sweden and UK) of the international collaborative Interphone study were included. Information on mobile phone use was obtained from interviews. Unconditional logistic regression was used with distance $<5 \mathrm{~cm}$ between tumour and mobile phone location (line from the corner of the mouth to the ear) as the outcome.

Results Majority of the gliomas were located in frontal and temporal lobes in both regular mobile phone users and never regular users of mobile phones ( $43 \%$ vs $37 \%$ in frontal and $30 \%$ vs $28 \%$ temporal lobe). The proportion of tumours with a distance $<5 \mathrm{~cm}$ between the putative source of exposure and mid-point of glioma was $22 \%$ among regular users (mean $6.3 \mathrm{~cm}$ ) and $24 \%$ among non-users (mean $6.2 \mathrm{~cm}$ ). Cumulative call-time (hours) and duration of use (years) were not associated with proximity of tumours to the source of exposure. Conclusion Tumour location is unaffected by mobile phone use.

\section{P2-20 WITHDRAWN}

\section{P2-21 ETHNIC INEQUALITIES IN MYOCARDIAL INFARCTION INCIDENCE, INTERVENTIONS AND SURVIVAL IN SCOTLAND: THE SCOTTISH HEALTH AND ETHNICITY LINKAGE STUDY (SHELS)}

doi:10.1136/jech.2011.142976h.57

${ }^{1} \mathrm{~N}$ Bansal, ${ }^{*} \mathrm{C}$ Fischbacher, ${ }^{1} \mathrm{R}$ Bhopal, ${ }^{1} \mathrm{H}$ Brown, ${ }^{1} \mathrm{M}$ Steiner, ${ }^{3} \mathrm{~S}$ Capewell. ${ }^{1}$ University of Edinburgh, Edinburgh, UK; ${ }^{2}$ Information Services Division, Edinburgh, UK; ${ }^{3}$ University of Liverpool, Liverpool, UK

Introduction Ethnic variations in coronary heart disease are large with a $50-70 \%$ excess consistently observed in South Asians. It is not clear whether this is attributable to increased incidence, poor survival, or both. We compared incidence and outcome of first acute myocardial infarction (AMI) by ethnic group in Scotland in relation to cardiac intervention uptake, socioeconomic factors and proximity to hospital.

Methods We used linkage methods to combine ethnicity data from those aged $\geq 30$ years of age in the 2001 Scottish Census with records of subsequent hospital discharges and deaths between 1 May 2001 and 30 April 2008. We compared incidence (death or discharge) and case fatality following first AMI by ethnic group using the White Scottish as the standard comparison population.

Results AMI incidence rates were highest among Pakistani and lowest for Chinese, Other White British and Other White ethnic groups. Adjustment for highest educational qualification attenuated differences between White Scottish and other White groups but did not fully explain the excess in the Pakistani group. Pakistani women had lower HRs for death after AMI partly explained by shorter travel time to hospital. We found no evidence for lower uptake of cardiovascular procedures in Indians and Pakistanis.

Conclusions The known elevated coronary heart disease risk in South Asians principally reflects increased incidence in Pakistanis emphasising the need for aggressive management of modifiable cardiovascular risk factors. Pakistani women were protected from case fatality in part by their closer proximity to hospital and not increased uptake of interventional procedures.

\section{P2-22 IS CESAREAN SECTION ASSOCIATED WITH AN INCREASED RISK FOR OBESITY AT ADULTHOOD? A BRAZILIAN COHORT STUDY}

doi:10.1136/jech.2011.142976h.58

${ }^{1} \mathrm{H}$ Goldani, ${ }^{2} \mathrm{H}$ Bettiol, ${ }^{4} \mathrm{M}$ Morais, ${ }^{3} \mathrm{~A}$ A M da Silva, ${ }^{1} \mathrm{M}$ Agranonik, ${ }^{1} \mathrm{M}$ Z Goldani, ${ }^{2} \mathrm{M}$ A Barbieri. ${ }^{1}$ Hospital de Clínicas de Porto Alegre, Porto Alegre, Brazil;; ${ }^{2}$ Faculdade de Medicina de Ribeirão Preto- Universidade de São Paulo, Ribeirão Preto, Brazil; ${ }^{3}$ Universidade Federal do Maranhão, São Luis, Brazil; ${ }^{4}$ Universidade Federal de São Paulo, São Paulo, Brazil

Introduction Obesity is worldwide epidemic and increase in cesarean section rates have occurred in parallel. Overweight children had a lower proportion of the genus Bifidobacterium spp. in their intestinal microflora during infancy. Infants born by cesarean section have less Bifidobacterium spp. as predominant microbiota.

Objective we hypothesised that infants born by cesarean section are more likely to develop obesity in adulthood.

Methods We carried out a newborn cohort study in Ribeirão Preto, Brazil, started in 1978. A randomised sample of 2057 subjects from the original cohort (6827 individuals) was reassessed in 2002. Some co-variables were collected after birth: type of delivery, birth weight, maternal smoking and maternal schooling. The data from subjects were obtained at the time of their return for evaluation at 24 years of age: body mass index (BMI), physical activity, subject smoking, and income in minimum wages. Obesity was considered when $\mathrm{BMI} \geq 30$. A Poisson multivariable model was performed aiming to determine the impact of cesarean section on BMI at adulthood. The model was adjusted for subject and maternal factors.

Results The rates of obesity in young adults born by cesarean section was $15.2 \%$ vs $10.4 \%$ in those born by vaginal delivery $(p=0.002)$ Subjects who were born by cesarean section had an increased significant risk [1.57 (1.23-2.02)] for obesity at adulthood after controlling

Conclusion We may hypothesise that the differences in intestinal flora related to type of delivery section may have a role on the epidemic obesity worldwide. 